\title{
Childhood Executive Functioning Inventory: Adaptação e Propriedades Psicométricas da Versão Brasileira
}

\author{
Bruna Tonietti Trevisan - Universidade Presbiteriana Mackenzie, São Paulo, Brasil \\ Natália Martins Dias - Centro Universitário FIEO, Osasco, Brasil \\ Arthur de Almeida Berberian - Centro Universitário FIEO, Osasco, Brasil \\ Alessandra Gotuzo Seabra - Universidade Presbiteriana Mackenzie, São Paulo, Brasil
}

\begin{abstract}
Resumo
O objetivo do estudo foi traduzir, adaptar e investigar propriedades psicométricas da Childhood Executive Functioning Inventory (CHEXI) em uma amostra de crianças brasileiras. Após tradução, adaptação transcultural, retrotradução e equivalência semântica, realizada por juízes da área, a versão brasileira da CHEXI foi respondida por pais e professores de 408 crianças, idades entre 4 e 7 anos, também avaliadas com a Escala de Maturidade Mental Colúmbia e SNAP-IV. Elevados índices de consistência interna foram encontrados. A análise fatorial exploratória gerou dois fatores para a versão brasileira: um fator mais geral de funções executivas e um específico de inibição. As pontuações nas subescalas de planejamento, regulação e, marginalmente, memória de trabalho da CHEXI explicaram de modo significativo o indicador de desatenção da SNAP-IV, enquanto a pontuação na subescala de inibição explicou o indicador de hiperatividade/impulsividade. Os resultados fornecem bons parâmetros psicométricos para a CHEXI, além de contribuir para a realização de estudos com funções executivas e indicadores de TDAH no país.

Palavras-chave: avaliação psicológica, neuropsicologia, função executiva, transtorno do déficit de atenção com hiperatividade
\end{abstract}

\section{Childhood Executive Functioning Inventory: adaptation and psychometric properties of the Brazilian version}

\begin{abstract}
The aim of the study was to translate, adapt and investigate psychometric properties of Childhood Executive Functioning Inventory (CHEXI) for a sample of Brazilian children. After translation, cultural adaptation, back-translation and semantic equivalence performed by area judges, the Brazilian version of CHEXI was answered by parents and teachers of 408 children aged between 4 and 7 years, also evaluated with the Columbia Mental Maturity Scale and SNAP-IV. High internal consistency indices were found. Exploratory factor analysis yielded two factors for the Brazilian version: a more general factor of executive functions and one specific factor of inhibition. The scores on the subscales of planning, regulation and marginally working memory in CHEXI explained significantly the inattention indicator of SNAP-IV, while the score on the inhibition subscale explained the indicator of hyperactivity/impulsivity. The results provide good psychometric parameters for CHEXI, besides contributing to the studies with Executive functions and ADHD signs in Brazil.

Keywords: psychological assessment, neuropsychology, executive function, attention deficit and hyperactivity disorder
\end{abstract}

\section{Childhood Executive Functioning Inventory: Adaptación y Propiedades} Psicométricas de la versión brasileña

\begin{abstract}
Resumen
El objetivo del estudio fue traducir, adaptar e investigar las propiedades psicométricas del Childhood Executive Functioning Inventory (CHEXI) en una muestra de niños brasileños. Después de la traducción, adaptación transcultural, retro-traducción y equivalencia semántica realizada por jueces del área, la versión brasileña de la CHEXI fue respondida por padres y maestros de 408 niños de edad comprendida entre 4 y 7 años, también evaluados con la Escala de Madurez Mental Columbia y SNAP-IV. Se encontraron altos índices de consistencia interna. El análisis factorial exploratorio generó dos factores para la versión brasileña: un factor más general de las funciones ejecutivas y un factor específico de inhibición. Las puntuaciones en las sub- escalas de planificación, regulación y marginalmente memoria de trabajo de la CHEXI explicaron significativamente el indicador de falta de atención de la SNAP-IV, mientras que la puntuación en la sub-escala de inhibición explicó el indicador de hiperactividad / impulsividad. Los resultados proporcionaron buenos parámetros psicométricos para la CHEXI, además de contribuir para la realización de estudios con funciones ejecutivas e indicadores de TDAH en el país.

Palabras-clave: evaluación psicológica; neuropsicología; función ejecutiva; Trastorno de Déficit de Atención con Hiperactividad
\end{abstract}

A avaliação das funções executivas (FE) tem recebido forte ênfase na literatura nacional em Neuropsicologia e Psicologia Cognitiva, resultado dos esforços e produções de grupos de pesquisa dedicados à área (Barros \& Hazin, 2013; Cardoso et al., 2010; Carreiro et al., 2014; Seabra \& Dias, 2012; Natale, Teodoro, 
Barreto, \& Haase, 2008). As funções executivas (FE) configuram um domínio cognitivo complexo relacionado com o estabelecimento de comportamentos dirigidos a metas predefinidas, envolvendo as habilidades de planejamento, flexibilidade mental, inibição e memória de trabalho (Miyake et al., 2000). São genericamente compreendidas como processos "de cima para baixo" (top-down) que possibilitam tanto a tomada de decisão (Willcutt, Doyle, Nigg, Faraone, \& Pennington, 2005) quanto o controle e a regulação dinâmica da cognição, com base em representações internas ou feedback ambiental (Barch, Braver, Carter, Poldrack, \& Robbins, 2009). Em conjunto, esses processos permitem ao indivíduo se adaptar a situações novas e a resolver problemas, de modo a alcançar objetivos.

Tradicionalmente, as FE têm sido avaliadas com recurso a testes de desempenho (Diamond, 2013), embora outros métodos ou ferramentas tenham sido sugeridos e possam complementar o processo de avaliação. Por exemplo, uma revisão recente mostrou que, para além dos testes padronizados, tem sido crescente o número de estudos e de instrumentos ecológicos e funcionais. Os instrumentos ecológicos buscam combinar procedimentos sistemáticos e padronizados de administração e pontuação em tarefas que simulam situações cotidianas. Por sua vez, instrumentos funcionais podem incluir observação, entrevista ou escalas que mensuram, por autorrelato ou relato de terceiros, o modo pelo qual $\mathrm{o}$ indivíduo desempenha determinadas tarefas em seu cotidiano (Zimmermann, Cardoso, Kochhann, Jacobsen, \& Fonseca, 2014).

Um exemplo de instrumento funcional é a Childhood Executive Functioning Inventory - CHEXI (Thorell \& Nyberg, 2008), que pode ser respondida por pais e professores e foi desenvolvida com foco na avaliação das FE em crianças com Transtorno do Déficit de Atenção/Hiperatividade (TDAH). Esse transtorno é caracterizado por padrão persistente de desatenção e/ou hiperatividade/impulsividade, mais frequente e grave do que o observado em indivíduos em níveis comparáveis do desenvolvimento (APA, 2013). É considerado um transtorno do desenvolvimento e tende a persistir durante muitos anos ou por toda a vida (Mattos, 2002; Schmitz, Polanczyk \& Rohde, 2007), com prevalência estimada de 5,29\% entre crianças e adolescentes até 18 anos de idade (Polanczyk et al., 2007). É reconhecido que déficits em FE são um dos componentes da complexa Neuropsicologia do TDAH, havendo evidências substanciais em relação a diferenças de estrutura, funcionamento e neuroquímica cerebrais no transtorno, em circuitos que são consideradas chave para as FE (Willcutt et al., 2005; Castellanos et al., 2006).

Em 1997, Barkley propôs uma teoria que sugere que crianças com TDAH deveriam apresentar primariamente dificuldades em controle inibitório. De fato, os modelos da década de 1990 e início dos anos 2000 sugeriam que déficits em aspectos da FE, mais do que déficits atencionais primários, representariam um fator nuclear do transtorno (Barkley, 1997). Nesse sentido, Barkley dividiu o controle inibitório (CI) em três diferentes funções: inibição de respostas preponderantes; habilidade de inibir respostas que já estão em curso; e controle de interferência. Do ponto de vista do processamento da informação, alterações em CI podem dificultar outros aspectos das FE, tais como iniciação de comportamento, planejamento, organização e flexibilidade cognitiva, memória de trabalho, autorregulação, que envolve motivação e estado de alerta, além da internalização do discurso e reconstituição (Berlin, Bohlin \& Rydell, 2003; Durstewitz, Seamans \& Sejnowski, 2000).

No entanto, nos últimos dez anos, estudos têm sugerido que déficits em FE não sejam os fatores causais em todos os casos de TDAH (Nigg, 2006). Nesse sentido, uma importante meta-análise da área (Willcutt et al., 2005) que cuidadosamente selecionou 83 estudos que investigaram FE em 3734 crianças com TDAH e 2969 crianças sem o transtorno, demostrou que o grupo com TDAH apresentou dificuldades principalmente em memória de trabalho, inibição, vigilância e planejamento. No entanto, os déficits apresentaram tamanho de efeito moderado e, ao mesmo tempo, nem todos os indivíduos com os sintomas clínicos de TDAH apresentam prejuízos em FE.

Assim, não há dados suficientes para garantir que déficits primários de FE causam o transtorno, porém, a relação entre TDAH e déficits em FE é bastante relatada na literatura, visto que déficits em inibição, especificamente na pré-escola (Berlin, Bohlin \& Rydell, 2003; Sonuga-Barke, Dalen \& Remington, 2002) e em outros aspectos das funções executivas na adolescência e vida adulta (Boonstra, Ossterlaan, Sergeant \& Buitelaar, 2005; Martel, Nikolas \& Nigg, 2007), são frequentes nessa população.

Devido à mencionada relação, mas possível dissociação com os sintomas clínicos, ao desenvolver os itens da CHEXI, o objetivo de seus autores foi fornecer exemplos específicos que demandem diferentes tipos de controle executivo em vez de situações gerais ou que estivessem relacionadas diretamente aos sintomas 
de TDAH. Em sua construção, os autores focalizaram especificamente comportamentos relacionados às FE, evitando assim, sobreposição semântica desnecessária entre déficits de FE e sintomas de TDAH. Os itens da CHEXI se agrupam nas subescalas de memória de trabalho, inibição, autorregulação e planejamento. As questões correspondentes aos itens do inventário foram criadas a partir do modelo de Barkley (1997), no qual controle inibitório, memória de trabalho e autorregulação são vistos como constituindo a maioria dos déficits de FE em crianças com TDAH. Os itens referentes à memória de trabalho, de modo mais específico, foram baseados na teoria de Baddeley e Hitch (1974).

Estudo prévio conduzido com a versão original do instrumento em amostra de crianças pré-escolares identificou que seus itens se agruparam em dois grandes fatores: Inibição, que agregou as subescalas de Inibição e Autorregulação e Memória de Trabalho, que incluiu as subescalas Memória de Trabalho e Planejamento. Essa solução fatorial se repetiu quando considerados os protocolos respondidos por pais e por professores. Além disso, o estudo evidenciou índice de fidedignidade por meio de procedimento de teste-reteste bastante satisfatório (Thorell \& Nyberg, 2008). Com base no achado dos dois fatores globais (memória de trabalho e inibição), uma investigação subsequente mostrou que ambos os fatores contribuem significativamente para diferenciar entre grupo de crianças com TDAH e controles, tanto quando considerado o relato de pais, quanto de professores e mesmo após controle da inteligência. Assim, o estudo revelou que a CHEXI é efetiva em discriminar, em uma amostra de crianças de cinco a oito anos, aquelas com diagnóstico de TDAH de controles com desenvolvimento normal, mostrando que o instrumento possui alta sensibilidade e especificidade (Thorell, Eninger, Brocki, \& Bohlin, 2010).

Dada a carência de instrumentos funcionais para avaliação das funções executivas no contexto nacional, o objetivo deste estudo foi traduzir, adaptar e investigar características psicométricas da versão brasileira da CHEXI. Este último objetivo inclui: a) analisar a precisão da CHEXI; b) investigar evidências de validade de construto; e c) investigar evidências de validade por relação com outras variáveis, verificando se o instrumento pode predizer os indicadores de desatenção e hiperatividade/impulsividade avaliados pela SNAP-IV. Uma vez verificadas tais propriedades psicométricas e disponibilizada a CHEXI no contexto nacional, o estudo poderá contribuir a futuras investigações na área, que terão ponto de partida para aprofundar averiguações do instrumento, podendo contribuir, inclusive, ao seu futuro uso no contexto clínico.

\section{Método}

\section{Descrição do Instrumento e Procedimento de Tradução e Adap- tação da CHEXI}

A CHEXI foi desenvolvida originalmente no idioma inglês por Thorell e Nyberg (2008) com o objetivo de avaliar FE em crianças com TDAH. O instrumento possui 26 itens, sendo que cada um deles é pontuado em uma escala Likert de cinco níveis (definitivamente falso, falso, parcialmente verdadeiro, verdadeiro, definitivamente verdadeiro). Os itens são agrupados em quatro subescalas: memória de trabalho (11 itens, e.g., "Tem dificuldade em entender instruções verbais a menos que também seja mostrado como fazer algo.”), planejamento (quatro itens, e.g., "Tem dificuldade com tarefas ou atividades que envolvam várias etapas.”), controle inibitório (seis itens, e.g. "Tem tendência para fazer coisas sem pensar primeiro no que poderia acontecer.") e autorregulação (cinco itens, e.g. "Tem clara dificuldade em fazer coisas que considera chatas.").

De acordo com as autoras, os itens do instrumento foram desenvolvidos de modo que retratassem exemplos específicos de comportamentos/situações que demandassem diferentes tipos de controle executivo, ou seja, os itens da CHEXI focalizam especificamente comportamentos relacionados às habilidades executivas e não sintomas de TDAH propriamente, o que é considerado por seus autores como uma limitação da maioria das escalas e inventários existentes. No entanto, cabe apontar que a ocorrência de alguma sobreposição entre itens da CHEXI e indicadores ou sintomas de TDAH é compreensível, dada a relação entre as habilidades avaliadas na escala e as manifestações clínicas do transtorno. $\mathrm{O}$ instrumento fornece uma medida funcional das habilidades executivas da criança e o tempo estimado para seu preenchimento é de 5 a 10 minutos. No presente estudo, a CHEXI foi aplicada tanto a pais quanto a professores. Para análise da precisão do instrumento, considerou-se os protocolos respondidos por pais e professores, separadamente; para investigação das evidências de validade analisou-se as repostas de pais e professores conjuntamente.

Para a tradução e adaptação do instrumento para a língua portuguesa no Brasil, inicialmente foram conduzidas três etapas para sua tradução, conforme descrito a seguir: 
1) A etapa 1 consistiu em duas traduções do instrumento original em inglês para o português brasileiro, realizadas de forma independente: a primeira delas, por um profissional especialista em inglês, e a segunda, por um neuropsicólogo fluente no idioma inglês;

2) A etapa 2 consistiu nas retrotraduções das traduções em português da etapa 1 para o inglês, realizadas por dois profissionais especialistas em inglês. As retrotraduções ocorreram de modo independente e foram feitas de forma cega com relação ao perfil dos profissionais da primeira etapa;

3) A etapa 3 consistiu na apreciação formal de equivalência semântica entre as retrotraduções e o original, e entre as duas versões das traduções e das retrotraduções.

4) Para a análise descrita na etapa 3, foi usado um formulário em que os significados dos termos e expressões de cada um dos itens, bem como os enunciados e os cinco níveis da escala Likert foram julgadas em três níveis: semelhante, aproximado e diferente. Isso foi feito comparando as duas versões (da tradução e da retrotradução), bem como retrotradução em relação ao original. Os resultados foram analisados de forma a desenvolver uma versão síntese. Essa etapa foi feita por profissionais não envolvidos nas etapas 1 e 2 e que dominam a área de avaliação neuropsicológica.

\section{Estudo das Características Psicométricas da CHEXI}

\section{Participantes}

Participaram 408 crianças, de amostra não clínica, com idades entre 4 e 7 anos $(M=5,51, D P=0,59)$, de ambos os sexos $(F=54,4 \%, M=46,6 \%)$, estudantes do Ensino Infantil I (44,1\%), Ensino Infantil II (10,5\%) e $1^{\circ}$ ano do Ensino Fundamental (45,3\%) de escolas municipais da Grande São Paulo, bem como seus professores e pais. Todas as crianças tinham percentis na Escala de Maturidade Mental Colúmbia acima de 25, conforme avaliação feita no presente estudo como critério de inclusão da amostra. Não houve qualquer outro critério de inclusão ou exclusão, exceto o consentimento livre e esclarecido. A Tabela 1 apresenta a caracterização dos participantes.

\section{Instrumentos}

Além da versão brasileira da CHEXI, foram utilizados os instrumentos descritos a seguir.
Escala de Maturidade Mental Columbia (EMMC)

A EMMC (Burgemeister, Blum \& Lorge, 1971) é um teste padronizado que avalia a aptidão geral de raciocínio de crianças entre $3 \mathrm{a} 6 \mathrm{~m}$ e $9 \mathrm{a} 11 \mathrm{~m}$ de idade (Alves \& Duarte, 2001). A criança deve observar pranchas com três a cinco desenhos cada e escolher qual desenho é diferente ou não se relaciona aos outros. A instrução especifica que a criança deve escolher a figura que não combina com as outras. Para tanto, a ela deve descobrir qual a regra subjacente à organização das figuras, permitindo-lhe excluir apenas uma.

\section{SNAP-IV}

A SNAP-IV é um questionário de domínio público, formulada a partir dos critérios do DSM-IV, objetivando avaliar sintomas do Transtorno de Déficit de Atenção/Hiperatividade. É composto pela descrição dos 18 sintomas, sendo nove de desatenção e nove de hiperatividade/impulsividade, os demais itens relacionados a comportamentos opositores-desafiadores não foram considerados nas análises. Para cada um dos itens é pontuado em uma escala de quatro níveis de gravidade (nem um pouco, só um pouco, bastante, demais) e pode ser respondido por pais e professores. A versão brasileira foi adaptada por Mattos et al. (2006). No presente estudo, os escores no instrumento serão considerados como indicadores de desatenção e/ou de hiperatividade/impulsividade, utilizados para investigação de padrões de convergência com a CHEXI.

\section{Procedimento}

O projeto foi submetido e aprovado pelo Comitê de Ética em Pesquisa. Após assinatura do Termo de Consentimento Livre e Esclarecido, as crianças foram avaliadas na EMMC, em sessão única com duração de aproximadamente 20 minutos, na própria escola e durante o período escolar regular. Pais e professores responderam a CHEXI e o SNAP-IV. O preenchimento de ambos ocorreu na ausência do aplicador, sendo os instrumentos preenchidos e devolvidos à escola das crianças participantes.

\section{Análise de Dados}

A precisão da CHEXI foi verificada por meio do alfa de Cronbach e método das metades de Spearman-Brown, considerando relatos de pais e professores separadamente. As análises subsequentes consideraram os relatos de pais e professores conjuntamente (média aritmética da pontuação atribuída por pais e professores à criança). A validade de construto foi 
Tabela 1

Caracterização dos Participantes

\begin{tabular}{|c|c|c|c|c|}
\hline \multirow{5}{*}{$1^{\circ}$ ano } & \multicolumn{2}{|r|}{ Idade } & \multicolumn{2}{|c|}{ Frequências (\%) } \\
\hline & Média & 6,01 & 4 anos & 3,7 \\
\hline & Desvio Padrão & 0,198 & 5 anos & 42,9 \\
\hline & Mínimo & 5 & 6 anos & 52,5 \\
\hline & Máximo & 7 & 7 anos & 1,0 \\
\hline \multirow{4}{*}{ Infantil I } & Média & 4,72 & & \\
\hline & Desvio Padrão & 0,591 & Feminino & 54,4 \\
\hline & Mínimo & 4 & Masculino & 46,6 \\
\hline & Máximo & 6 & & \\
\hline \multirow{4}{*}{ Infantil II } & Média & 5,21 & $1^{\circ}$ ano & 44,1 \\
\hline & Desvio Padrão & 0,405 & Infantil I & 10,5 \\
\hline & Mínimo & 5 & Infantil II & 45,3 \\
\hline & Máximo & 6 & & \\
\hline \multirow{4}{*}{ Total } & Média & 5,51 & & \\
\hline & Desvio Padrão & 0,586 & & \\
\hline & Mínimo & 4 & & \\
\hline & Máximo & 7 & & \\
\hline \multirow{5}{*}{$\begin{array}{l}\text { EMMC } \\
\text { Total }\end{array}$} & & EMMC & & \\
\hline & Média & 66,49 & & \\
\hline & Desvio Padrão & 20,744 & & \\
\hline & Mínimo & 25 & & \\
\hline & Máximo & 99 & & \\
\hline
\end{tabular}

investigada por meio de análise fatorial exploratória, com rotação oblimin e evidências de validade por relação com outras variáveis foi investigada por meio de análise de regressão linear múltipla, tendo as subescalas da CHEXI como variáveis preditoras e os índices de desatenção e hiperatividade da SNAP-IV como variáveis critério. Utilizou-se método Enter para inserção das variáveis no modelo.

\section{Resultados}

Inicialmente, foram conduzidas análises descritivas dos desempenhos nos instrumentos. O desempenho na SNAP-IV obteve média 5,89 $(D P=4,98)$ para o escore em desatenção e média 8,11 $(D P=5,78)$ para o escore em hiperatividade, ambos respondidos pelos pais. Em relação ao mesmo instrumento respondido pelos professores, foi verificada média $6,07(D P=6,41)$ para os itens que avaliação desatenção e média 4,78 $(D P=6,38)$ para os itens que avaliam hiperatividade. O desempenho na CHEXI respondida pelos pais obteve as seguintes médias: $61,46(D P=17,32)$ para o escore total; 20,48 $(D P=6,38)$ para o escore em memória de trabalho; 9,04 $(D P=3,30)$ para planejamento; $14,24(D P=4,05)$ em regulação e $16,95(D P=4,58)$ em controle inibitório. E, o desempenho na CHEXI respondida pelos professores obteve as seguintes médias: $57,79(D P=$ $21,76)$ para o escore total; $20,43(D P=8,41)$ para o escore em memória de trabalho; 9,10 $(D P=3,66)$ para planejamento; $11,95(D P=4,68)$ em regulação e 14,18 $(D P=5,38)$ em controle inibitório.

Em seguida, investigou-se a precisão da CHEXI. A análise revelou índices elevados, com alfa de Cronbach de 0,94 para CHEXI administrado aos pais, e 0,98 para os professores. O coeficiente de Spearman-Brown foi de 0,92 e 0,96 para pais e professores, respectivamente.

Para as análises subsequentes, as respostas de pais e de professores foram consideradas conjuntamente. A fim de investigar evidências de validade de construto, foi conduzida análise fatorial exploratória. A análise 
encontrou dois fatores para a CHEXI, que explicam $76,92 \%$ da variância total. Essas informações constam na Tabela 2.

A Tabela 3 a seguir apresenta a distribuição das cargas fatoriais dos itens da CHEXI, considerando as cargas iguais ou superiores a 0,30 . É possível observar a presença de dois fatores, sendo que o primeiro caracteriza um fator mais geral de funções executivas, enquanto o segundo apresenta itens das subescalas de regulação e controle inibitório e apenas um item da subescala de memória de trabalho.

De forma a buscar evidências de validade por relação com outras variáveis, verificando se a pontuação na CHEXI pode predizer os indicadores de TDAH avaliados pela SNAP-IV, foi conduzida análise de regressão linear múltipla. Para tanto, foram usadas as subescalas teóricas da CHEXI (regulação, memória de trabalho, controle inibitório e planejamento) como variáveis preditoras e os escores em desatenção e em hiperatividade/impulsividade na SNAP-IV como variáveis dependentes.

O modelo revelou três habilidades executivas que explicaram $66,7 \%$ da variabilidade em desatenção. Ainda considerando desatenção, planejamento e regulação, alcançaram uma contribuição significativa ( $p$ $<0.02$ ), enquanto memória de trabalho alcançou um nível marginal de significância $(p<0.06)$. Por sua vez, considerando os indicadores de hiperatividade/impulsividade, o controle inibitório foi a única habilidade executiva que alcançou nível significativo $(\phi<0.001)$, explicando $61 \% \mathrm{da}$ variabilidade no indicador.

\section{Discussão}

O objetivo do estudo foi traduzir, adaptar e investigar características psicométricas da CHEXI no contexto brasileiro. Consoante à primeira etapa desse objetivo, foram realizadas a tradução, retrotradução, comparação entre versões, adaptação e fechamento da versão brasileira da CHEXI. Essa primeira parte do estudo resultou em um instrumento funcional para avaliação das $\mathrm{FE}$ em crianças, ferramenta que, até a presente data, não se encontra disponível no contexto nacional, apesar de trabalhos de grupos de pesquisa com outros instrumentos, a exemplo do Behavior Rating Inventory of Executive Function - BRIEF (Carim, Miranda, \& Bueno, 2012). Tais ferramentas e os estudos de suas características psicométricas podem ter relevância à área dada a ausência de instrumentos funcionais para avaliação de FE no Brasil e a relevância desse tipo de mensuração para complementar o processo de avaliação neuropsicológica (Zimmermann et al., 2014).

Após conclusão da versão brasileira da CHEXI, a segunda etapa do estudo foi investigar as características psicométricas do instrumento. Inicialmente, a precisão da CHEXI foi investigada por meio do coeficiente alfa de Cronbach e do método das metades de Sperman -Brown. Os resultados apontam para a alta precisão e consistência interna da versão brasileira do CHEXI para avaliação de FE em crianças, tanto por meio do relato de pais quanto pelo relato de professores. Essa etapa é importante uma vez que o conteúdo dos itens pode ser fonte geradora de erros de medida, a exemplo de itens mal construídos (ou mesmo mal traduzidos/adaptados no caso do presente estudo) ou que não possam ser compreendidos com clareza pelos respondentes. Ainda, erros de mensuração podem estar atrelados a itens específicos ao longo do teste. Por exemplo, algum item pode divergir ou explorar conteúdos distintos daqueles explorados pelos demais no teste, seja refletindo problemas na sua construção, seleção, entre outras. Assim, a fidedignidade é um parâmetro fundamental à averiguação da qualidade de um teste e, para alguns autores, pode ser tomada como evidência mínima para obtenção de uma medida válida de comportamento (AERA, APA; NCME, 1999; Urbina, 2007). Nesse sentido, a versão brasileira da CHEXI mostrou-se altamente consistente e relativamente livre de erros de medida.

Tabela 2

Resultados da Análise Fatorial Exploratória da CHEXI

\begin{tabular}{cccc}
\hline & \multicolumn{3}{c}{ Eigenvalues iniciais } \\
\cline { 2 - 4 } & Total & \% de Variância Explicada & \% Cumulativa \\
\hline 1 & 18,113 & 69,664 & 69,664 \\
2 & 1,886 & 7,255 & 76,919 \\
\hline
\end{tabular}


Tabela 3

Cargas Fatoriais dos Itens da CHEXI

\begin{tabular}{|c|c|c|}
\hline \multirow{2}{*}{ Item } & \multicolumn{2}{|c|}{ Componente } \\
\hline & 1 & 2 \\
\hline 1. Tem dificuldade em lembrar instruções longas. & 0,893 & \\
\hline 2. Raramente consegue se motivar a fazer algo que não queira. & 0,498 & 0,461 \\
\hline 3. Tem dificuldade em lembrar o que está fazendo quando está no meio de uma atividade. & 0,893 & \\
\hline $\begin{array}{l}\text { 4. Tem dificuldade para dar continuidade em tarefas menos interessantes, a menos que tenha } \\
\text { sido prometido algum tipo de recompensa pra fazer tal tarefa. }\end{array}$ & 0,461 & 0,490 \\
\hline 5. Tem tendência para fazer coisas sem pensar primeiro no que poderia acontecer. & 0,402 & 0,530 \\
\hline 6. Quando lhe pedem para fazer várias coisas, ele(a) lembra-se somente da primeira ou da última. & 0,752 & \\
\hline $\begin{array}{l}\text { 7. Tem dificuldade em encontrar uma forma diferente para resolver um problema quando ele(a) } \\
\text { fica sem saída. }\end{array}$ & 0,787 & \\
\hline 8. Quando algo precisa ser feito, ele(a) frequentemente se distrai com algo mais interessante. & 0,401 & 0,541 \\
\hline 9. Esquece com facilidade o que pediram para ele(a) buscar. & 0,983 & \\
\hline $\begin{array}{l}\text { 10. Fica excessivamente empolgado quando algo especial está para acontecer (por exemplo, } \\
\text { viajar, ir para uma festa). }\end{array}$ & & 0,788 \\
\hline 11. Tem clara dificuldade em fazer coisas que considera chatas. & & 0,763 \\
\hline $\begin{array}{l}\text { 12. Tem dificuldade em planejar uma atividade (por exemplo, lembrar-se de trazer tudo o que for } \\
\text { necessário para uma viagem ou para a escola) }\end{array}$ & 0,760 & \\
\hline 13. Tem dificuldade em permanecer na sua atividade, mesmo quando é dito para ele(a) fazer isso. & 0,371 & 0,610 \\
\hline $\begin{array}{l}\text { 14. Tem dificuldade em realizar atividades que exijam várias etapas (por exemplo, para crianças } \\
\text { pequenas, se vestirem completamente sem lembretes; para crianças mais velhas, fazer toda a } \\
\text { lição de casa de forma independente). }\end{array}$ & 0,722 & \\
\hline 15. Para conseguir se concentrar, ele(a) deve achar a tarefa interessante. & 0,507 & 0,411 \\
\hline 16. Tem dificuldade em conter o riso ou risada em situações em que é inadequado. & & 0,857 \\
\hline $\begin{array}{l}\text { 17. Tem dificuldade em contar uma história sobre algo que aconteceu de modo que os outros } \\
\text { possam compreender com facilidade. }\end{array}$ & 0,961 & \\
\hline $\begin{array}{l}\text { 18. Tem dificuldade de parar imediatamente uma atividade quando é dito para fazer isso. Por } \\
\text { exemplo, ele (a) precisa pular algumas vezes a mais ou jogar no computador um pouco mais } \\
\text { mesmo após ter sido pedido para parar. }\end{array}$ & & 0,724 \\
\hline $\begin{array}{l}\text { 19. Tem dificuldade em compreender instruções verbais a menos que também seja mostrado } \\
\text { como fazer algo. }\end{array}$ & 0,869 & \\
\hline 20. Tem dificuldade com tarefas ou atividades que envolvam várias etapas. & 0,983 & \\
\hline 21. Tem dificuldade em antecipar ou aprender a partir da experiência. & 0,886 & \\
\hline $\begin{array}{l}\text { 22. Age de um modo mais desenfreado ou sem limites quando comparado(a) com outras } \\
\text { crianças em um grupo (por exemplo, em uma festa de aniversário ou durante uma atividade em } \\
\text { grupo). }\end{array}$ & & 0,984 \\
\hline $\begin{array}{l}\text { 23. Tem dificuldade em realizar coisas que exijam esforço mental, como contar de trás para } \\
\text { frente. }\end{array}$ & 0,899 & \\
\hline 24. Tem dificuldade em manter as coisas em mente quando está fazendo outra atividade. & 0,833 & \\
\hline 25. Pensa em voz alta, mesmo quando realiza tarefas relativamente simples. & 0,400 & 0,546 \\
\hline $\begin{array}{l}\text { 26. Tem dificuldade em compreender o conceito de tempo em comparação com colegas da } \\
\text { mesma idade. }\end{array}$ & 0,758 & \\
\hline
\end{tabular}


Outro parâmetro psicométrico fundamental é a validade (AERA, APA; NCME, 1999; Urbina, 2007). O estudo investigou evidências de validade de construto e por relação com outras variáveis para a versão brasileira da CHEXI. A análise fatorial exploratória a partir dos itens da CHEXI retornou uma solução com dois fatores. De fato, estudo prévio (Thorell \& Nyberg, 2008) também encontrou dois fatores, ou seja, o fator Inibição, que agregou as subescalas de inibição e regulação e o fator Memória de Trabalho, que incluiu as subescalas memória de trabalho e planejamento, solução fatorial que se repetiu tanto quando consideradas respostas de pais quanto dos professores. Apesar da convergência no achado dos dois fatores, a distribuição e cargas fatoriais observadas nesta investigação não foram as mesmas do estudo original. Assim, como esperado, o primeiro fator agrupou todos os itens das subscalas Memória de Trabalho e Planejamento, porém, também teve cargas fatoriais de quatro itens da subescala Regulação (itens 2, 4, 8 e 15, com cargas fatoriais divididas entre os fatores 1 e 2 de modo relativamente equivalente) e dois itens da subescala Inibição (itens 5 e 13, com cargas fatoriais maiores no fator 2). Desse modo, esse primeiro fator parece ser mais um fator geral de FE do que de memória de trabalho propriamente, como evidenciado por Thorell e Nyberg (2008) e replicado posteriormente por Thorell et al. (2010).

Algumas hipóteses podem ser lançadas na tentativa de explicar a diferença observada na composição fatorial da versão brasileira da CHEXI. Uma delas remete a aspectos do desenvolvimento. Por exemplo, nos estudos de Thorell e Nyberg (2008) e de Thorell et al. (2010), as crianças mais jovens de suas amostras possuíam cinco anos, enquanto que no presente estudo foram incluídas crianças de quatro anos. Estudos têm apontado para uma maior relação entre as FE em idades precoces, com maior diferenciação das distintas habilidades com a progressão da idade (Wiebe, Sheffield, Nelson, Clark, Chevalier \& Espy, 2011). Assim, o fato de possuir crianças mais jovens na amostra pode ser repercutido em maior relação/menor diferenciação entre as habilidades avaliadas, conduzindo ao fator 1 , um fator mais geral de FE. Também o fato de, neste estudo, terem sido utilizados os escores conjuntos de pais e professores (enquanto nos estudos originais utilizou-se escores independentes dessas fontes) deve ser lembrado. Estudos futuros deverão considerar as questões aqui levantadas.

Já o segundo fator apresentou-se de modo mais consistente com o esperado a partir do estudo original com a CHEXI. Ou seja, esse fator agrupou todos os itens das subscalas de inibição e regulação, aproximando-se do fator Inibição evidenciado por Thorell e Nyberg (2008). Apenas um item da subscala teórica de memória de trabalho (item 25 - Pensa em voz alta, mesmo quando realiza tarefas relativamente simples) apresentou carga nesse fator. Tal fato, porém pode estar atrelado à carga de regulação subjacente ao item. Pensar em voz alta ou falar consigo mesmo enquanto realiza uma atividade é uma estratégia para regular a própria conduta (Dawson \& Guare, 2010). Assim, apesar de originalmente ser considerado um item da subescala de Memória de Trabalho, é plausível que o item também possua uma carga de regulação, o que explicaria o fato de ter carregado em ambos os fatores.

Por fim evidências de validade por relação com outras variáveis foram investigadas tendo como critério indicadores de desatenção e de hiperatividade/ impulsividade. As pontuações nas subescalas de planejamento, regulação e, marginalmente, memória de trabalho, foram capazes de predizer de modo significativo o indicador de desatenção avaliado pela SNAP-IV, enquanto a pontuação na subescala de inibição explicou de modo significativo o indicador hiperatividade. Esses achados, mesmo em amostra não clínica, corroboram a relação entre FE e os indicadores do transtorno (Willcutt et al., 2005; Castellanos et al., 2006). De modo geral, as relações estabelecidas entre FE e indicadores do TDAH eram esperadas e foram consistentes com a literatura, por exemplo, Willcutt et al. (2005) também identificou, porém em grupo que comtemplava crianças com diagnóstico do transtorno, que habilidades de memória de trabalho, inibição e planejamento estavam entre as mais comprometidas em indivíduos com TDAH. De modo específico, porém, notou-se que diferentes habilidades contribuíram a indicadores específicos. Assim, indicadores de desatenção foram mais bem explicados pela habilidade de planejamento e regulação, e secundariamente pela memória de trabalho. Conforme apresentado por Diamond (2005), de fato a desatenção tem como déficit cognitivo principal alterações na memória de trabalho, o que pode levar a variações nos tempos de reação e dificuldade na manutenção de um nível adequado de engajamento ao longo de tarefas de alta demanda, consideradas por Seargent, Geurts, Huijbregts, Scheres e Oosterlaan (2003) como medida de regulação.

Por outro lado, indicadores de hiperatividade/ impulsividade foram explicados unicamente pela inibição. De fato, segundo Diamond (2005), a alteração em 
inibição é o principal déficit relacionado à hiperatividade/impulsividade, sendo que alterações em controle inibitório estão entre os achados mais consistentes em estudos sobre FE e TDAH (Nigg, 2001). Para Barkley (1997), a inibição inclui, além do controle de interferência, a capacidade de inibir respostas preponderantes, ou seja, automáticas, e respostas em curso. Nesse sentido, um indivíduo mais hábil em inibir um comportamento inadequado seria, de fato, avaliado como possuindo menores indicadores de hiperatividade/impulsividade.

Apesar de resultados satisfatórios, elencou-se algumas limitações do estudo: a ausência de participantes de amostra clínica e de dados de precisão teste-reteste que, por um lado, permitiria verificar a estabilidade da resposta dos participantes ao longo do tempo e, por outro, respaldaria o uso da CHEXI em ensaios clínicos. Como o conceito de validade está relacionado a níveis, ou seja, um instrumento pode ter mais ou menos evidências de validade dependendo do acúmulo de diversas fontes (Urbina, 2007), o presente estudo acumulou duas fontes de evidências de validade: construto e por relação com outras variáveis, tendo a SNAP como fonte de indicadores de TDAH, além de duas fontes de precisão (consistência interna e método das metades). Nesse sentido, são necessários novos estudos para maior acúmulo de evidências de validade da CHEXI para uso clínico. Como terceira limitação, sabe-se da diversidade demográfica entre as regiões brasileiras e também do fato de não sido feito estratificação demográfica que siga as características médias brasileiras. A amostra, ainda que contemple 408 crianças de 4 a 7 anos de idade, é uma amostra não probabilística, por conveniência e oriunda do Estado de São Paulo. Finalmente, como uma última limitação cabe mencionar a ausência de critérios de exclusão para condições clínicas que poderiam interferir nas pontuações da CHEXI. No entanto, sobre essa questão, cabe mencionar que a amostra do estudo apresenta maior representatividade externa.

Novos estudos são necessários a fim de ampliar evidências de validade da versão brasileira da CHEXI, incluindo estudos de sua sensibilidade e especificidade na identificação de indivíduos com transtornos que co-ocorrem com déficits de FE, delimitação de pontos de corte ou elaboração de normas e investigação de sua estrutura fatorial e utilidade clínica em diferentes faixas etárias. Ainda, considerando o objetivo original dos autores e para o qual o instrumento foi desenvolvido, ou seja, avaliar FE em crianças com TDAH (Thorell \& Nyberg, 2008), é possível que uma adequação de seus itens (ou desenvolvimento de novos) seja necessária, uma vez que outros modelos teóricos elencam habilidades relevantes à compreensão da Neuropsicologia do transtorno e que não são contemplados na CHEXI, a exemplo da aversão à demora (Sonuga-Barke, 2003; 2005) e da regulação do estado (Sergeant, 2000; 2005). A partir desse primeiro estudo com a CHEXI, novas investigações estão sendo conduzidas buscando uma integração desses modelos teóricos na construção de um instrumento que abarque de forma mais ampla as alterações de FE.

\section{Considerações Finais}

A versão brasileira da CHEXI mostrou dados satisfatórios de fidedignidade, revelando-se com alta consistência interna. O modelo fatorial do estudo original não foi replicado. $\mathrm{O}$ instrumento apresentou composição bifatorial, com um primeiro componente mais global (subescalas de memória de trabalho e planejamento e alguns itens de inibição e regulação) e um segundo componente de inibição (subescalas inibição e regulação). Tal achado pode estar atrelado a questões desenvolvimentais, embora aspectos culturais também devam ser considerados. Estudos deverão aprofundar a compreensão dos itens específicos que carregaram em ambos os fatores de modo a entender o que de fato o item mensura. Itens reelaborados ou mesmo novos itens poderão constituir a versão final desse instrumento. Evidências de validade por relação com outras variáveis foram obtidas, ilustrando o papel preditor das FE sobre indicadores de desatenção e hiperatividade/ impulsividade mesmo em amostra não clínica.

\section{Referências}

AERA, APA, \& NCME (1999). Standards for educational and psychological testing. Washington, D.C. Author.

American Psychiatric Association. (2002). Manual diagnóstico e estatístico de transtornos mentais ( $4^{\mathrm{a}} \mathrm{ed}$.). Porto Alegre: Artmed.

Baddeley, A. D., \& Hitch, G. (1974). Working memory. Em G. H. Bower (Ed.), The psychology of learning and motivation: Advances in research and theory (pp. 47-89). New York: Academic Press.

Barch, D. M., Braver, T. S., Carter, C. S., Poldrack, R. A, \& Robbins, T. W. (2009). CNTRICS final task selection: Executive control. Schizophrenia Bulletin, 35(1), 115-35. doi: 10.1093/schbul/sbn154 
Barkley, R. A. (1997). ADHD and the nature of self-control. New York: Guilford.

Barros, P. M., \& Hazin, I. (2013). Avaliação das funções executivas na infância: Revisão dos conceitos e instrumentos. Psicologia em Pesquisa, 7(1), 13-22. doi: 10.5327/Z1982-1247201300010003

Berlin, L., Bohlin, G., \& Rydell, A. M. (2003). Relations between inhibition, executive functioning, and ADHD-symptoms: A longitudinal study from age 5 to 81/2. Child Neuropsychology, 9, 255-266. doi: 10.1076/chin.9.4.255.23519

Boonstra, A. M., Oosterlaan, J., Sergeant, J. A., \& Buitelaar, J. K. (2005). Executive Functioning in Adult ADHD: A Meta-Analytic Review. Psychological Medicine, 35(8), 1097-1108. doi: 10.1017/ S003329170500499X

Burgemeister, B., Blum, L., \& Lorge, I. (1971). Columbia Mental Maturity Scale. Nova York: Harcourt, Brace, \& Jovanovich.

Cardoso, C. D. O., Carvalho, J. C. N., Cotrena, C., Bakos, D. D. G. S., Kristensen, C. H., \& Fonseca, R. P. (2010). Estudo de fidedignidade do instrumento neuropsicológico Iowa Gambling Task. Jornal Brasileiro de Psiquiatria, 59(51), 279-285. doi: 10.1590/ S0047-20852010000400003

Carim, D. B., Miranda, M. C., \& Bueno, O. F. A. (2012). Tradução e adaptação para o português do Behavior Rating Inventory of Executive Function - BRIEF. Psicologia: Reflexão e Crítica, 25(4), 653661. doi: 10.1590/S0102-79722012000400004

Carreiro, L. R. R., Dias, N. M., Malloy-Diniz, L. F., Trevisan, B. T., Minervino, O, C. A. S. M., Roazzi, A., \& Seabra, A. G. (2014). Testes de funções executivas no Brasil. Em A. G. Seabra, J. A. Laros. E. C. Macedo, N. Abreu. (Eds.), Inteligência e funcões executivas: avanços e desafios para a avaliação neuropsicológica (pp.84-111). São Paulo: Memnon.

Castellanos, F. X., Sonuga-Barke, E. J. S., Milham, M. P., \& Tannock., R. (2006). Characterizing cognition in ADHD: Beyond executive dysfunction. Trends in Cognitive Science, 10(3), 117-123. doi:10.1016/j. tics.2006.01.011

Dawson P, \& Guare R. (2010). Executive skills in children and adolescents: A practical guide to assessment and intervention. New York: The Guilford Press.
Diamond, A. (2013). Executive functions. Annual Review of Psychology, 64, 135-168. doi: 10.1146/ annurev-psych-113011-143750

Durstewitz, D., Seamans, J. K., \& Sejnowski, T. J. (2000). Neurocomputational models of worling memory. Nature Neuroscience. 3, 1184-1191. doi: $10.1038 / 81460$

Martel, M., Nikolas, M., \& Nigg, J. T. (2007). Executive functions in adoloscents with ADHD. Jornal Am Acad Child Adolesc Psychiatry, 46(11), 1437-1444. doi: 10.1097/chi.0b013e31814cf953

Mattos, P. (2002). Avaliação neuropsicológica do TDAH. Em E.C, Macedo, M. J.Gonçalves, F. C. Capovilla \& A. L. Sennyey (Eds.). Tecnologia em (re) habilitação cognitiva. São Paulo, SP: Edunisc.

Mattos, P. Serra-Pinheiro, M. A., Rohde, L. A., \& Pinto, D. (2006). Apresentação de uma versão em português para uso no Brasil do instrumento MTA-SNAP-IV de avaliação de sintomas de transtorno do déficit de atenção/hiperatividade e sintomas de transtorno desafiador e de oposição. Revista de Psiquiatria, 28(3), 290-7. doi: 10.1590/ S0101-81082006000300008

Miyake, A., Friedman, N. P., Emerson M. J., Witzki, A. H., Howerter, A., \& Wager T. D. (2000) The unity and diversity of executive functions and their contributions to complex "Frontal Lobe" tasks: a latent variable analysis. Cognitive psychology, 41, $49-100$

Natale, L. L., Teodoro, L. M., \& Haase, B. (2008). Propriedades psicométricas de tarefas para avaliar funções executivas em pré- escolares. Psicologia em Pesquisa, 2(2), 23-35. Recuperado de http:// pepsic.bvsalud.org/scielo.php?pid $=$ S1982-12472008000200004\&script $=$ sci_arttext

Nigg, J. T. (2001). Is ADHD a disinhibitory disorder? Psychological Bulletin,127(5), 571. Recuperado de http://www.ncbi.nlm.nih.gov/pubmed/11548968

Nigg, J. T. (2006). What Causes ADHD? Understanding what goes wrong and why. New York: The Guildford Press.

Polanczyk, G., Lima, M. S., Horta, B. L., Biderman, J., \& Rohde, L. A., (2007). The worldwide prevalence of ADHD: A systematic review and metaregression analysis. The American Journal of Psychiatry, 164(6), 
942-948. Recuperado de http://www.ncbi.nlm. nih.gov/pubmed/17541055

Schmitz, M., Polanczyk, G., \& Rohde, L. A. P. (2007). TDAH: Remissão na adolescência e preditores de persistência em adultos. Jornal brasileiro de psiquiatria, 56(1), 25-29. Recuperado de http://www.scielo. br/pdf/jbpsiq/v56s1/a06v56s1.pdf

Seabra, A. G., \& Dias, N.M. (Eds.). (2012). Avaliação Neuropsicológica Cognitiva: Atenção e funções executivas. (1a. ed.). São Paulo: Memnon.

Sergeant, J. A., Geurts, H., Huijbregts, S., Scheres, A., \& Oosterlaan, J. (2003). The top and the bottom of ADHD: A neuropsychological perspective. Neuroscience \& Biobehavioral Reviews, 27, 583-592. Recuperado de http://www.ncbi.nlm.nih.gov/ pubmed/14624803

Sonuga-Barke, E. J. S. (2005). Causal models of attention-deficit/ hyperactivity disorder: From common simple deficits to multiple developmental pathways. Biological Psychiatry, 57, 1231-1238. Recuperado de http://www.ncbi.nlm.nih.gov/ pubmed/15949993

Sonuga-Barke, E. J. S. (2003). The dual pathway model of $\mathrm{AD} / \mathrm{HD}$ : An elaboration of neuro-developmental characteristics. Neuroscience and Biobehavioral Reviews, 27, 593-604. Recuperado de http://www. ncbi.nlm.nih.gov/pubmed/14624804

Sonuga-Barke, E., J. S., Dalen, L., Daley, D., \& Remington, B. (2002). Are planning, working memory, and inhibition associated with individual differences in preschool ADHD symptoms? Developmental neuropsychology, 21(3), 255-272. Recuperado de http:// www.ncbi.nlm.nih.gov/pubmed/12233938
Thorell, L. B., \& Nyberg, L. (2008). The childhood executive functioning inventory (CHEXI): A new rating instrument for parents and teachers. Developmental Neuropsychology, 33, 536-552. doi: 10.1080/87565640802101516.

Thorell, L. B., Eninger, L., Bricki, K. C., \& Gunilla, B. (2010). Childhood executive function inventory (CHEXI): A promising measure for identifying young children with ADHD? Journal of Clinical and Experimental Neuropsychology, 32(1), 38-43. doi: 10.1080/13803390902806527

Urbina, S. (2007). Introdução aos testes psicológicos e seus usos. Em S. Urbina (Ed.), Fundamentos da testagem moderna (pp. 11-41). Porto Alegre: Artmed.

Wiebe, S. Sheffield, T., Nelson, J., Clark, C., Chevalier, N., \& Espy, K. A. (2011). The structure of executive function in 3-year-olds. Journal of Experimental Child Psychology, 108(3), 436-452. doi: 10.1016/j.jecp.2010.08.008

Willcutt, E. G., Doyle, A. E., Nigg, J. T., Faraone, S. V, \& Pennington, B. F. (2005). Validity of the Executive Function Theory of Attention- Deficit / Hyperactivity Disorder: A Meta-Analytic Review. Biological Psychiatry, 1;57(11): 1336-46. doi: 10.1016/j.biopsych.2005.02.006

Zimmermann, N. C. C.O., Kochhann, R., Jacobsen, G., \& Fonseca, R. P. (2014). Contributions of the ecological approach to the neuropsychology of executive functions. Temas em Psicologia, 22(3), 639654. doi: 10.9788/TP2014.3-09

Recebido em: 12/06/2015

Reformulação: 30/10/2015

Aceito em: 25/11/2015 
Sobre os autores:

Bruna Tonietti Trevisan é Psicóloga, Mestre, Doutora e Pós-doutoranda em Distúrbios do Desenvolvimento pela Universidade Presbiteriana Mackenzie (UPM). Pesquisadora do Grupo de Neuropsicologia Infantil da UPM (www.facebook.com/neuropsiinfantil).

E-mail: brunattrevisan@gmail.com

Natália Martins Dias é Psicóloga, Mestre e Doutora (pós-doutorada) em Distúrbios do Desenvolvimento pela Universidade Presbiteriana Mackenzie (UPM). Docente do Programa de Pós-Graduação Stricto Sensu em Psicologia Educacional do Centro Universitário FIEO (UniFIEO), coordenadora do Grupo de Investigação em Neuropsicologia, Desenvolvimento e Educação (GINDE) da UniFIEO (www.facebook.com/gindefieo) e pesquisadora do Grupo de Neuropsicologia Infantil da UPM.

E-mail: natalia_mdias@yahoo.com.br

Arthur A. Berberian é Psicólogo, Doutor em Psiquiatria e Psicologia Médica pela Universidade Federal de São Paulo (Unifesp). Docente do Programa de Pós-Graduação Stricto Sensu em Psicologia Educacional do Centro Universitário FIEO (UniFIEO). Membro do Lab Interdisciplinar de Neurociências Clínicas (LiNC) - Unifesp.

E-mail: arthur.berberian@gmail.com

Alessandra Gotuzo Seabra é Psicóloga pela Universidade de São Paulo, Mestre e Doutora com Pós-doutorado em Psicologia (Psicologia Experimental) pela Universidade de São Paulo. Docente do Programa de Pós-Graduação em Distúrbios do Desenvolvimento da Universidade Presbiteriana Mackenzie. Coordenadora do Grupo de Neuropsicologia Infantil (www.facebook.com/neuropsiinfantil).

E-mail: alessandragseabra@gmail.com

Contato com os autores:

UNIFIEO - Fundação Instituto de Ensino para Osasco

Av. Franz Voegeli, 300 - Continental

Osasco-SP, Brasil

CEP: 06020-190 